\title{
Graphene-based resonant-tunneling structures
}

\author{
J. Milton Pereira, Jr. ${ }^{\text {a) }}$ \\ Department of Physics, University of Antwerp, Groenenborgerlaan 171, B-2020 Antwerpen, Belgium \\ and Departamento de Fisica, Universidade Federal do Ceara, Fortaleza, Ceara 60455-760, Brazil \\ P. Vasilopoulos \\ Department of Physics, Concordia University, 7141 Sherbrooke Ouest, Montreal, Quebec H4B 1R6, Canada \\ F. M. Peeters \\ Department of Physics, University of Antwerp, Groenenborgerlaan 171, B-2020 Antwerpen, Belgium
}

(Received 7 September 2006; accepted 21 February 2007; published online 30 March 2007)

\begin{abstract}
Resonant electronic transmission through graphene-based double barriers (wells) is studied as a function of the incident wave vector, the widths and heights (depths) of the barriers (wells), and the separation between them. Resonant features in the transmission result from resonant electron states in the wells or hole states in the barriers and strongly influence the ballistic conductance of the structures. (C) 2007 American Institute of Physics. [DOI: 10.1063/1.2717092]
\end{abstract}

Recently graphene and graphene-based microstructures have been realized experimentally. They exhibit unusual properties and have potential technological applications especially as an alternative to the current Si-based technology. The relativisticlike properties of carriers in graphene, ${ }^{1-4}$ such as an unusual quantum Hall effect, ${ }^{5}$ result from the gapless and approximately linear electron spectrum near the Fermi energy at two inequivalent points of the Brillouin zone. The charge carriers in these structures are described as massless, chiral "relativistic" fermions, governed by the Dirac equation, and their relativistic behavior is expected to lead to the observation of perfect transmission across potential barriers, known as the Klein tunneling. ${ }^{6}$ In addition, very recently electronic confinement was demonstrated in graphene microstructures using standard lithography methods. ${ }^{7}$ Recent studies have considered tunneling through single graphene barriers, ${ }^{8}$ wells, ${ }^{9}$ and $n-p$ - $n$ junctions. ${ }^{10}$ One important aspect of the electronic transport in quantum structures in graphene, that so far has not been investigated, is resonance effects on the transmission. In this letter we investigate them as a function of the barrier (well) height (depth) and separation, and contrast those for double barriers with those for "nonrelativistic" electrons.

The crystal structure of undoped graphene layers is that of a honeycomb lattice of covalent-bond carbon atoms. To each carbon atom corresponds a valence electron and the structure is composed of two sublattices, labeled A and B. The low-energy excitations of the system in the vicinity of the $\mathbf{K}$ point and in the presence of a potential $U$ are described by the two-dimensional (2D) Dirac equation,

$$
\left\{v_{F}[\boldsymbol{\sigma} \cdot \hat{\mathbf{p}}]+m v_{F}^{2} \sigma_{z}\right\} \Psi=(E-U) \Psi,
$$

where the pseudospin matrix $\vec{\sigma}$ has components given by Pauli's matrices, $\hat{\mathbf{p}}=\left(p_{x}, p_{y}\right)$ is the momentum operator. The "speed of light" of the system is $v_{F}$, i.e., the Fermi velocity $\left(v_{F} \approx 1 \times 10^{6} \mathrm{~m} / \mathrm{s}\right)$. The eigenstates of Eq. (1) are twocomponent spinors $\Psi=\left[\psi_{A}, \psi_{B}\right]^{T}$, where $\psi_{A}$ and $\psi_{B}$ are the envelope functions associated with the probability amplitudes at the respective sublattice sites of the graphene sheet.

\footnotetext{
${ }^{a)}$ Electronic mail: joaomilton.pereira@ua.ac.be
}

The term $\propto m v_{F}^{2}$ creates an energy gap and may arise due to an interaction with a substrate.

In the presence of a one-dimensional confining potential $U=U(x)$, we attempt solutions of Eq. (1) in the form $\psi_{A}(x, y)=\phi_{A}(x) e^{i k_{y} y}$ and $\psi_{B}(x, y)=i \phi_{B}(x) e^{i k_{y} y}$ because of the translational invariance along the $y$ direction. The resulting coupled, first-order differential equations for $\phi_{A}(x)$ and $\phi_{B}(x)$ can be easily decoupled. They read as

$$
\frac{d^{2} \phi_{C}}{d \xi^{2}}+\left(\Omega^{2}-\beta^{2}\right) \phi_{C}-\frac{u^{\prime}}{\Omega_{ \pm}}\left( \pm \frac{d \phi_{C}}{d \xi}-\beta \phi_{C}\right)=0 .
$$

Here $\xi=x / L, \quad \Omega_{ \pm}=\epsilon-u \pm \Delta, \quad \Omega=\left(\Omega_{+} \Omega_{-}\right)^{1 / 2}, \quad \beta=k_{y} L, \quad u$ $=U L / \hbar v_{F}, \epsilon=E L / \hbar v_{F}$, and $\Delta=m v_{F} L / \hbar ; L$ is the width of the structure, $u^{\prime}$ the derivative of $u$ with respect to $\xi$, and the $+(-)$ sign refers to $C=A(C=B)$.

For a single quantum well, with a square potential of height $U_{0}$, Eq. (2) admit solutions which describe electron states confined across the well and propagating along it. ${ }^{9}$ For confined states, the spinor components decay exponentially in the region $\xi<-1 / 2$. Then $\phi_{A}(x)$ can be written ${ }^{9}$ as

$$
\begin{aligned}
\phi_{A}(x) & =e^{i \alpha \xi}+B_{1} e^{-i \alpha \xi}, \quad \xi<-1 / 2, \\
& =A_{2} e^{i \kappa \xi}+B_{2} e^{-i \kappa \xi}, \quad-1 / 2 \leqslant \xi \leqslant 1 / 2, \\
& =A_{3} e^{i \alpha \xi}, \quad \xi>1 / 2,
\end{aligned}
$$

where $\alpha=\left[\left(\epsilon-u_{0}\right)^{2}-\beta^{2}-\Delta^{2}\right]^{1 / 2}$ and $\kappa^{2}=\epsilon^{2}-\beta^{2}-\Delta^{2}$. Notice that $\phi_{A}(x)$ depends on $k_{y}$ through $\beta=k_{y} / L$. A similar expression holds for $\phi_{B}$. Then, the transmission coefficient is obtained as $T=\left|A_{3}\right|^{2}$ and $A_{3}$ is determined by matching $\phi_{A}$ and $\phi_{B}$ at $\xi=-1 / 2$ and $\xi=1 / 2$. This procedure can be repeated for double wells or barriers (with $\kappa \rightarrow-\kappa$ ) to obtain the transmission $T$ through them. We avoid giving any expressions for $T$ and instead concentrate on the results for it. We obtain them without the mass term since $\Delta$ is usually very small.

A $\left(k_{y}, k_{x}\right)$ contour plot of the logarithm of the transmission $T$ through a double barrier is shown in Fig. 1. Typical values for the barrier height $U_{0}=50 \mathrm{meV}$, the barrier width $L=50 \mathrm{~nm}$, and the interbarrier separation $d=100 \mathrm{~nm}$ were used. As seen, $T$ depends on the direction of propagation or incident angle $\theta\left(\tan \theta=k_{y} / k_{x}\right)$ and its overall behavior is 


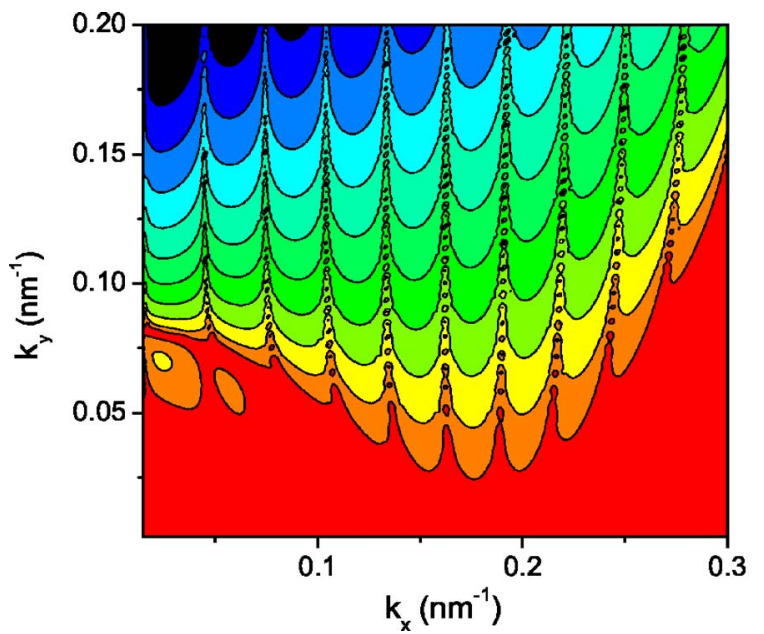

FIG. 1. Contour plot of the electronic transmission through a double barrier for $U_{0}=50 \mathrm{meV}$, width $L=50 \mathrm{~nm}$, and interbarrier separation $d=100 \mathrm{~nm}$.

similar to the earlier single-barrier $\operatorname{transmission}^{8}$ with the exception of well-defined resonances. The overall directional dependence of $T$ results from the chiral nature of the quasiparticles in graphene and a resonance caused by confined hole states in the barrier. Notice the perfect transmission $T$ $=1$ for normal or near-normal incidence $(\tan \theta \approx 0)$, which is a signature of Klein tunneling. ${ }^{8}$ Resonant transmission, which is typical for ordinary double-barrier structures, ${ }^{11}$ is found for values of $k_{x}$ that are integer multiples of $\pi / d$.

Another way of presenting the transmission is to plot $T$ versus the angle $\theta$ for fixed energy, as shown in Fig. 2 for $E=72,78$, and $85 \mathrm{meV}$ by the red-dashed, black-solid, and blue-dotted curves, respectively. As seen, the transmission is perfect not only for normal incidence but also for particular incidence angles, for which the values of the momentum components match the resonance peaks shown in Fig. 1.

It is interesting to explore the transmission as a function of the interbarrier separation $d$. A $\left(d, k_{y}\right)$ contour plot is shown in Fig. 3 for $U_{0}=100 \mathrm{meV}, E=27 \mathrm{meV}$, and $L$ $=50 \mathrm{~nm}$. Notice that for small values of $k_{y}$, i.e., small incidence angles, the transmission is perfect regardless of the magnitude of $d$. For larger $k_{y}$ the transmission oscillates as a function of the interbarrier separation with a period that depends on the momentum. This is a new feature, absent from

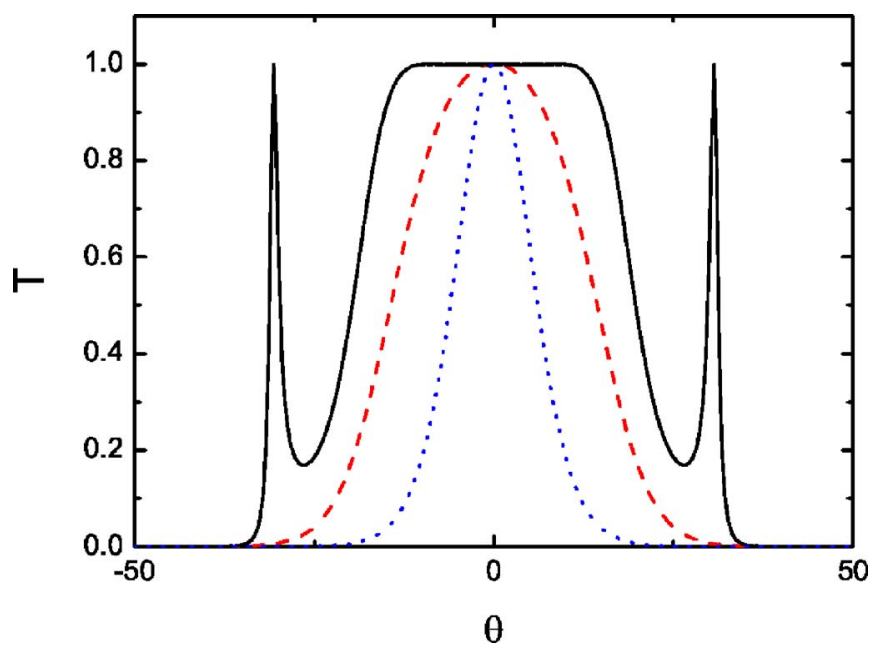

FIG. 2. Transmission through a double barrier as a function of the angle of incidence for $U_{0}=100 \mathrm{meV}, L=50 \mathrm{~nm}$, and interbarrier separation

$d=100 \mathrm{~nm}$.
Downloaded 05 Apr 2007 to 143.129.131.61. Redistribution subject to AlP license or copyright, see http://apl.aip.org/apl/copyright.jsp

$d=100 \mathrm{~nm}$.
Downloaded 05 Apr 2007 to 143.129.131.61. Redistribution subject to AlP license or copyright, see http://apl.aip.org/apl/copyright.jsp

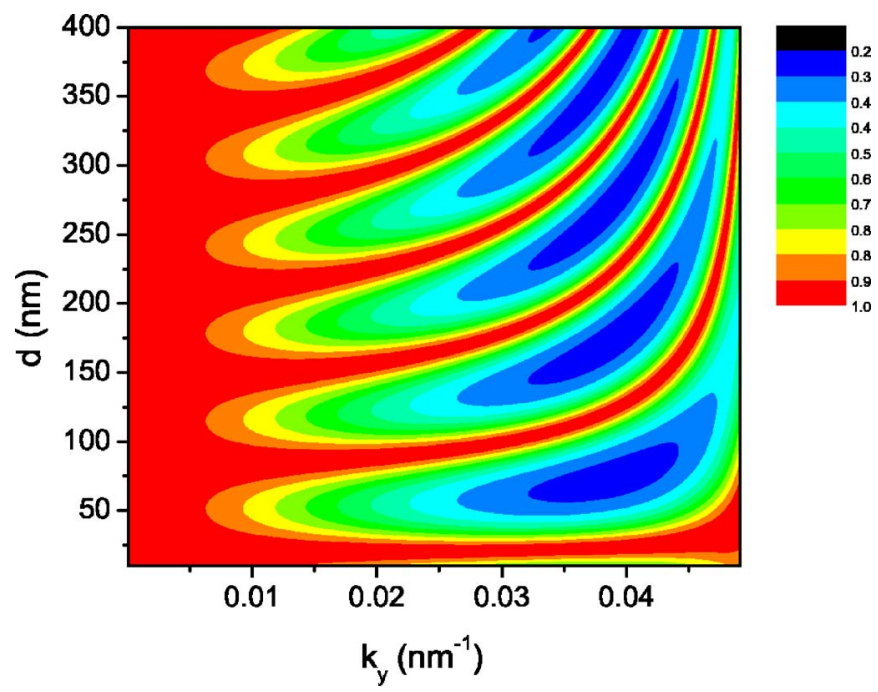

FIG. 3. $\left(d, k_{y}\right)$ contour plot of the transmission through a double barrier for $U_{0}=100 \mathrm{meV}, E=27 \mathrm{meV}$, and $L=50 \mathrm{~nm}$.

ordinary resonant structures, in which $T$ is independent of $k_{y}$.

The result for the transmission of electrons, with energies above a single well, was given in Ref. 9 and that for a double well is shown in Fig. 4. Though some features are qualitatively similar in both cases, that for a double well is characterized by a strong enhancement of the perfect transmission regions and has many resonant features that are absent from that for a single well. As compared to the doublebarrier case, cf. Fig. 1, the resonances are much broader and the transmission on the average much higher.

Selecting the wave vector components $k_{x}$ and $k_{y}$ is possible using quantum point contacts. However, experimentally one usually measures the "average" transmission. That is, one usually measures the current $J$ which is proportional to a weighted integral of the transmission $T\left(k_{x}, k_{y}\right)$. Repeating the argument of Ref. 11 for three-dimensional electrons with a parabolic energy spectrum, we find that for the linear spectrum $E=\hbar v_{F} k$ the current $J$, due to a voltage drop $e V$ along the $x$ direction, is given by $\left(\lambda=2 e v_{F} / h^{2}\right)$

$$
J=-\lambda \int T(E, \theta)[f(E)-f(E+e V)] E d E \cos \theta d \theta,
$$

where $f(E)$ is the Fermi function. For low temperatures we can approximate $[f(E)-f(E+e V)]$ by $-e V \delta\left(E-E_{F}\right)$ and ex-

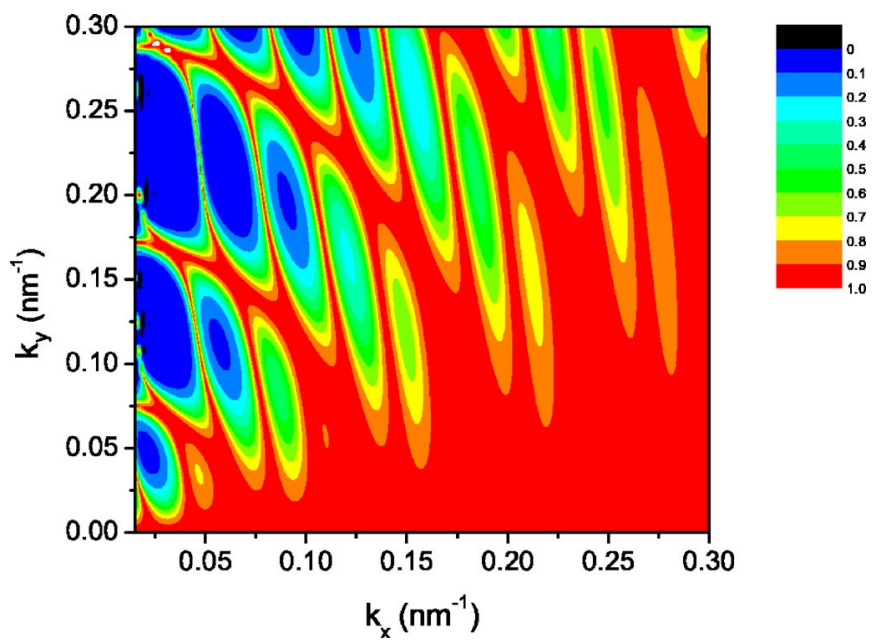

FIG. 4. As in Fig. 1 but for a double well with energy $E>U_{0}$, jsp 


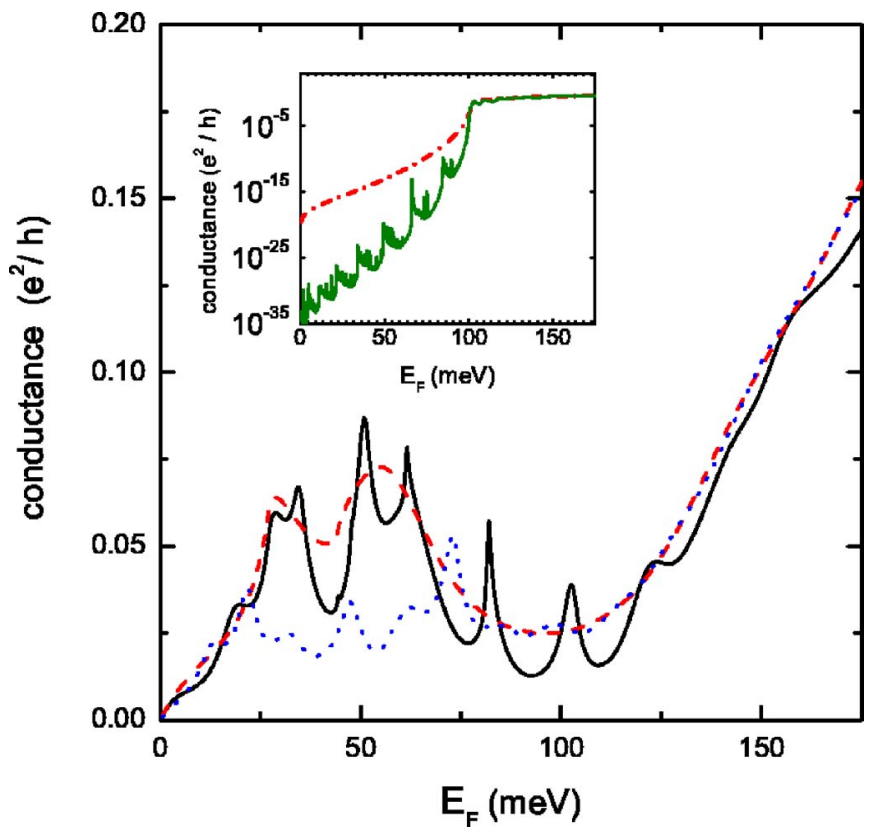

FIG. 5. Conductance through a single and double barrier vs energy $E$ for $\Delta=0$. Inset: conductance through the same structures for $m v_{F}^{2}=270 \mathrm{meV}$ : the red (green) curve is for a single (double) barrier.

tract the low-bias conductance from Eq. (4). For a 2D parabolic spectrum $\lambda$ is replaced by $2 e \sqrt{2 m} / h^{2}$ and $E d E$ by $E^{1 / 2} d E$ in Eq. (5). The main difference between the two cases is that in the latter the transmission $T(E, \theta)$ depends only on the magnitude of the $x$ component of the momentum, whereas in the former it depends on both components.

In Fig. 5 we plot the conductance versus the Fermi energy for a single-barrier (dashed curve) and for a doublebarrier structure for symmetric (solid curve) and asymmetric (dotted curve) barriers with width $L=50 \mathrm{~nm}$. The height of the first barrier is $U_{0}=100 \mathrm{meV}$ and that of the second $U_{0}=100,50$, and $0 \mathrm{meV}$, for the solid, dashed, and dotted curves, respectively. The interbarrier separation is $d=50 \mathrm{~nm}$. For energies below the maximum barrier height, the transmission is dominated by the Klein tunneling effect, in which the incident electrons are resonantly transmitted via the confined hole states in the barriers, resulting in peaks in the conductance. An additional pronounced structure is also found for double barriers due to an additional resonance effect caused by the quasiconfined electron states in the well region. This resonant structure is most explicit for symmetric barriers. One striking consequence of that is the fact that the low-bias conductance can be significantly lowered as the height of one of the barriers is decreased. As the Fermi energy increases above the maximum barrier height (here $100 \mathrm{meV}$ ) the resonant features practically disappear. In contrast with the $\Delta \neq 0$ results shown in the inset, which are a good qualitative approximation of the parabolic spectrum case, the results for graphene, in the limits $E_{F} \rightarrow 0$ and $E_{F} \rightarrow \infty$, show $T=1$ for near-normal incidence, see, e.g., Fig. 1. Using Eq. (4) we find immediately $G \propto E_{F}$ which agrees with our numerical results of Fig. 5. The present results are valid only in the ballistic regime, but in the presence of disorder the results for the conductance near the Dirac point are expected to be strongly modified.

In Fig. 6 we plot the conductance versus the Fermi energy for a single well (dashed curve) and for a double well,

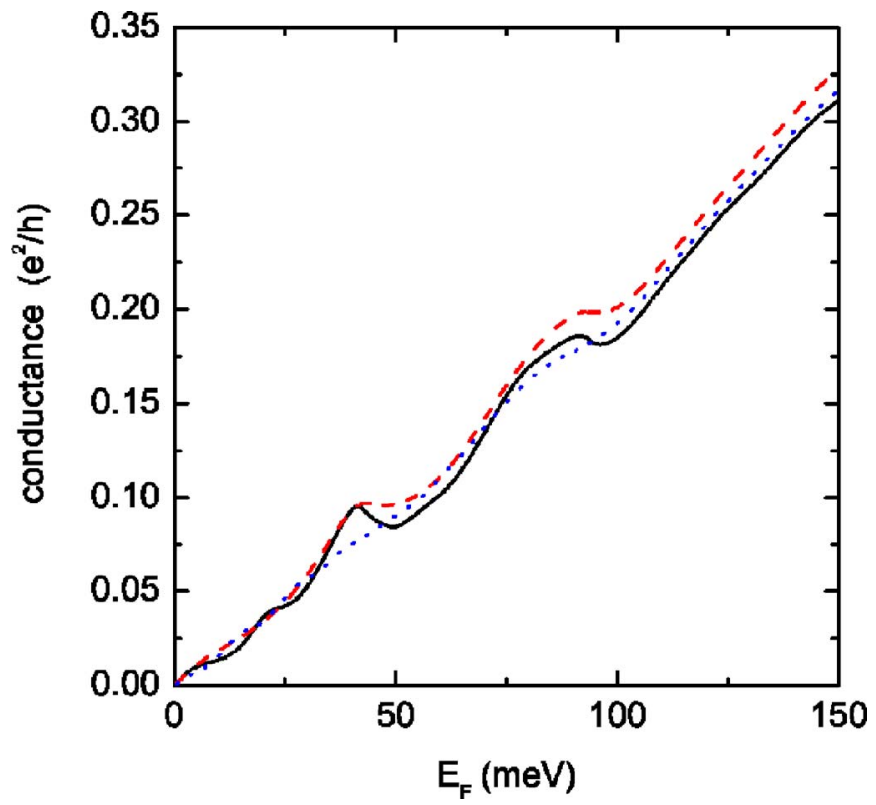

FIG. 6. Conductance through a single and double well vs Fermi energy $E_{F}$. The parameters are given in the text.

symmetric (solid curve) and asymmetric (dotted curve). The well width is $50 \mathrm{~nm}$. The depth of the first well is $U_{0}=100 \mathrm{meV}$ and that of the second $U_{0}=100,50$, and $0 \mathrm{meV}$, for the solid, dashed, and dotted curves, respectively. The interwell separation is $d=50 \mathrm{~nm}$. In this case, only weak resonant features are found that are determined completely by the first well. The presence of the second well almost does not influence the conductance.

In summary, we reported unusual resonant-tunneling features in graphene microstructures and a strong directional character of the transmission through them. The latter is perfect for a range of incident angles. No such behavior is found in the case of a parabolic spectrum. The conductance displays several resonant features that can be tuned by the barrier heights as well as by the separation between the barriers.

This work was supported by the Brazilian Council for Research $(\mathrm{CNPq})$, the Flemish Science Foundation (FWOVl), the Belgian Science Policy (IUAP), and the Canadian NSERC Grant No. OGP0121756.

${ }^{1}$ Y. Zheng and T. Ando, Phys. Rev. B 65, 245420 (2002).

${ }^{2}$ K. S. Novoselov, A. K. Geim, S. V. Morozov, D. Jiang, Y. Zhang, S. V. Dubonos, I. V. Grigorieva, and A. A. Firsov, Science 306, 666 (2004).

${ }^{3}$ Y. Zhang, Y. W. Tan, H. L. Stormer, and P. Kim, Nature (London) 438 , 201 (2005).

${ }^{4}$ V. P. Gusynin and S. G. Sharapov, Phys. Rev. Lett. 95, 146801 (2005).

${ }^{5}$ K. S. Novoselov, A. K. Geim, S. V. Morozov, D. Jiang, M. I. Katsnelson, I. V. Grigorieva, S. V. Dubonos, and A. A. Firsov, Nature (London) 438, 197 (2005)

${ }^{6}$ O. Klein, Z. Phys. 53, 157 (1929).

${ }^{7}$ C. Berger, Z. Song, X. Li, X. Wu, N. Brown, C. Naud, D. Mayou, T. Li, J. Hass, A. N. Marchenkov, E. H. Conrad, P. N. First, and W. A. de Heer, Science 312, 1191 (2006).

${ }^{8}$ M. I. Katsnelson, K. S. Novoselov, and A. K. Geim, Nat. Phys. 2, 620 (2006).

${ }^{9}$ J. Milton Pereira Jr., V. Mlinar, F. M. Peeters, and P. Vasilopoulos, Phys. Rev. B 74, 045424 (2006).

${ }^{10}$ V. V. Cheianov and V. I. Fal'ko, Phys. Rev. B 74, 041403 (2006).

${ }^{11}$ D. K. Ferry and S. M. Goodnick, Transport in Nanostructures (Cambridge University Press, New York, 2001). 